\title{
Generalized King linearity and new physics searches with isotope shifts
}

\author{
Julian C. Berengut $\odot,{ }^{1, *}$ Cédric Delaunay, ${ }^{2, \dagger}$ Amy Geddes, ${ }^{1, \ddagger}$ and Yotam Soreq $\oplus^{3, \S}$ \\ ${ }^{1}$ School of Physics, University of New South Wales, Sydney, New South Wales 2052, Australia \\ ${ }^{2}$ Laboratoire d'Annecy-le-Vieux de Physique Théorique LAPTh, CNRS - Université Savoie Mont Blanc, \\ BP 110, F-74941 Annecy-le-Vieux, France \\ ${ }^{3}$ Physics Department, Technion-Israel Institute of Technology, Haifa 3200003, Israel
}

(Received 25 May 2020; accepted 15 December 2020; published 31 December 2020)

\begin{abstract}
Atomic spectral lines for different isotopes are shifted, revealing a change in the properties of the nucleus. For spinless nuclei such isotope shifts for two distinct transitions are expected to be linearly related, at least at leading order in a change of the nuclear mass and charge distribution. Looking for a breaking of linearity in so-called King plots was proposed as a novel method to search for physics beyond the standard model. In the light of the recent experimental progress in isotope shift spectroscopy, the sensitivity of these searches will become limited by the determination of the isotope masses and/or by nuclear effects which may induce nonlinearities at an observable level. In this work, we propose two possible generalizations of the traditional King plot that overcome these limitations by including additional isotope shift measurements, thus significantly extending the new physics reach of King plots in a purely spectroscopy-driven approach.
\end{abstract}

DOI: 10.1103/PhysRevResearch.2.043444

\section{INTRODUCTION}

Fundamental interactions of the known elementary particles are well described by the standard model (SM) of particle physics. This theory has been tested up to TeV-scale energies by the ATLAS and CMS experiments conducted at the Large Hadron Collider (LHC).

Despite its great experimental success, the SM cannot be a complete theory of Nature. First of all, it does not account for observations related to the matter-antimatter asymmetry of the universe, neutrino oscillations and dark matter. Moreover, the SM seems incomplete also from a theoretical standpoint. Most importantly, it suffers from hierarchy problems related to the instability of the electroweak scale and the absence of $\mathrm{CP}$ violation in strong interactions. The SM also brings to light a deep flavor puzzle indicated by a very broad spectrum of elementary particle masses. All these considerations call for a completion of the SM, in the form of new interactions and particles.

Unfortunately, none of the above shortcomings of the SM unarguably points towards a preferred theoretical construct, nor even a specific energy scale for such a new physics (NP). For many decades, strongly motivated by the electroweakscale instability problem, physics beyond the SM (BSM) was

\footnotetext{
*julian.berengut@unsw.edu.au

†cedric.delaunay@lapth.cnrs.fr

‡a.geddes@unsw.edu.au

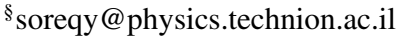

Published by the American Physical Society under the terms of the Creative Commons Attribution 4.0 International license. Further distribution of this work must maintain attribution to the author(s) and the published article's title, journal citation, and DOI. believed to emerge only at or slightly above the $\mathrm{TeV}$ scale. Today, the lack of evidence from the LHC program for NP at high-energies challenges this paradigm. Interestingly, in the meantime, new BSM theories have been found where the aforementioned problems of the SM are solved by much lighter new particles, with mass well below the GeV scale and small couplings to the matter fields [1-10].

This wide window of theoretical possibilities greatly motivates broad searches for NP at very different energy scales. While the accelerator-based program searches for NP at the high-energy frontier, precision measurements at low energies form a highly valuable complementary approach. See Refs. [11-13] for reviews. Low-energy measurements indirectly probe NP whose existence may be revealed either by comparing the SM predictions to the experimental results or by searching for signatures forbidden by (approximate) symmetries of the SM. Well known examples are the searches for electric dipole moments of elementary particles [14,15] or precision measurements of their magnetic dipole moments [16] and that of atomic parity-violating transitions [17,18].

Atomic spectral lines, and their isotope shifts (IS), are also extremely well measured. Yet, their calculation is not possible with comparable accuracy, except in very simple atoms like hydrogen, deuterium and helium or in purely leptonic bound states like positronium or muonium (for related work see, e.g., Refs. [19-21]). For heavier elements, a promising approach [22] consists in combining precision measurements of IS in so-called King plots [23] and testing for King linearity. In this approach, current IS spectroscopy may already probe new spin-independent interactions between electrons and neutrons at an unprecedented level [24,25]. Moreover, the sensitivity in probing NP with IS is expected to greatly improve in the near future as indicated by recent experimental developments. For instance, IS spectroscopy reached an accuracy of better than 
$10 \mathrm{~Hz}$ for electric quadrupole transitions in $\mathrm{Ca}^{+}$ions [26]. Also, a novel strategy of IS measurement was proposed [27] which is based on preparing pairs of different isotopes in an entangled state that oscillates at the IS frequency. By significantly improving on noise reduction, this technique allowed IS measurements at the $10 \mathrm{mHz}$ level. While this very high reach in accuracy was first demonstrated with $\mathrm{Sr}$ isotopes, it can in principle be implemented in other elements relatively easily.

As the accuracy of IS measurements keeps on improving, the potential to probe NP with King linearity will be curbed by two possible limitations. First, the uncertainty associated with nuclear masses will eventually exceed the IS measurement uncertainty. The King plot analysis is constructed in terms of so-called modified IS, which are IS frequencies divided by the difference of (inverse) isotope masses. Therefore nuclear masses must be known also with a very high precision. Typically, the relative mass uncertainty will become a limiting factor whenever it is comparable or larger than that of the IS measurement multiplied by the relative mass difference. For instance, assuming state-of-the-art mass determination $[28,29]$, mass uncertainties of ytterbium $(\mathrm{Yb})$ isotopes are expected to limit King linearity tests once the precision in IS measurements goes below the $\mathrm{Hz}$ level.

The second limitation comes from nonlinearities (NLs) of the King plot due to higher order nuclear corrections in the $\mathrm{SM}$. Linearity is the result of an approximate factorization of the electronic and nuclear effects in the two dominant contributions to the IS. This factorization is expected to break once subleading nuclear effects are included, which thus induces King linearity violation, namely NLs in King plots. Such NLs are expected to depend on the element and are challenging to calculate theoretically. Nevertheless, their size has been estimated in a few cases. For example, NLs were found to be rather small (below the $\mathrm{Hz}$ level) in $\mathrm{Ca}^{+}$but those could reach up to $\mathcal{O}(10) \mathrm{kHz}$ in King plots based on $\mathrm{Yb}^{+}$clock transitions [30] or fine-structure transitions in argon ions [31], for example. With a state-of-the-art accuracy in IS measurements at or even below the $\mathrm{Hz}$ level for some of these transitions, subleading nuclear effects will soon become an important limitation in the search for NP.

Very recently, dedicated NP searches with IS spectroscopy have been reported in $\mathrm{Yb}^{+}$[32] and $\mathrm{Ca}^{+}$[33], which constitute a significant improvement upon the prior most accurate test of King linearity performed with dipole transitions in $\mathrm{Ca}^{+}$ measured at a precision of $\mathcal{O}(100) \mathrm{kHz}$ [34]. The authors of Ref. [32] constructed a King plot from two quadrupole transitions in $\mathrm{Yb}^{+}\left({ }^{2} S_{1 / 2} \rightarrow{ }^{2} D_{3 / 2}\right.$ and $\left.{ }^{2} S_{1 / 2} \rightarrow{ }^{2} D_{5 / 2}\right)$ for which IS were measured with an accuracy of $\mathcal{O}(300) \mathrm{Hz}$. The resulting King plot shows an evidence of NL at the three standard deviation level. Such NL may result from NP or higher order SM contributions. With only ytterbium data, the two interpretations cannot be distinguished. In addition, the current accuracy of these measurement also indicates that the uncertainty due to the isotope mass determination already plays a role in the sensitivity to NP with the King analysis. In the meantime, the authors of Ref. [33] measured the $3 d{ }^{2} D_{3 / 2} \rightarrow$ ${ }^{2} D_{5 / 2}$ fine-structure transition in $\mathrm{Ca}^{+}$with $\mathcal{O}(20) \mathrm{Hz}$ accuracy and constructed a King plot by combining it with measurements of the ${ }^{2} S_{1 / 2} \rightarrow{ }^{2} D_{5 / 2}$ transition. The resulting King plot is consistent with linearity and has a comparable sensitivity to $\mathrm{NP}$ as the $\mathrm{Yb}^{+}$analysis of Ref. [32]. Therefore, as illustrated in Fig. 2 below, the NP explanation of the $\mathrm{Yb}^{+}$data appears in tension with the $\mathrm{Ca}^{+}$data, thus favoring a SM origin for the non linear behavior reported in Ref. [32].

In this paper we show how to overcome the limitations due to too large isotope mass uncertainties and/or higher order SM contributions without resorting to improved nuclear mass determination, nor theory calculations of nuclear NLs. Basically, we propose to use one (or more) additional IS transition(s) in order to eliminate the nuclear parameter(s) whose knowledge is not sufficiently accurate to allow for NP searches driven only by very precise IS spectroscopy. Reference [35] pointed out that it is possible to keep improving the NP sensitivity even in the presence of NLs by constructing a generalized King plot where the subleading nuclear physics parameters are absorbed by including IS measurements in three or more transitions. Here we further develop the generalized King plot analysis of Ref. [35] and show the potential improvement sensitivity to NP beyond the leading order contributions. Below, in order to illustrate the above proposal, we mostly focus on the case of one sub-leading SM effect and show the potential improvement.

The rest of the paper is organized as follows. Section II contains a review of the King analysis. In Sec. III, we present a modified King analysis without the input of isotope masses. A parametrization of higher order SM effects is presented Sec. IV. We show a generalization of the King analysis which include higher order effects in Sec. V. We demonstrate the above two methods in Sec. VI by examination of the ytterbium case. Finally, we conclude in Sec. VII.

\section{REVIEW OF KING LINEARITY AND ITS BREAKING}

Consider a set of atomic transitions $i$ and their IS frequencies $v_{i}^{a} \equiv v_{i}^{A_{0}}-v_{i}^{A}$ between two isotopes of mass number $A_{0}$ and $A$, with $a \equiv A_{0} A{ }^{1}{ }^{1}$ The latter is usually understood as being comprised of two SM terms [23] and possibly one NP term [22],

$$
v_{i}^{a}=K_{i} \mu_{a}+F_{i} \delta\left\langle r^{2}\right\rangle_{a}+\alpha_{\mathrm{NP}} X_{i} \gamma_{a} .
$$

The first term is the mass shift (MS), with $\mu_{a} \equiv 1 / m_{A_{0}}$ $1 / m_{A}$ being the difference of inverse nuclear masses, and the second term is the volume or field shift (FS). The third term in Eq. (1) is the result of NP, where $\alpha_{\mathrm{NP}}$ denotes the NP coupling strength, while $X_{i}$ and $\gamma_{a}$ are the NP electronic constant and nuclear parameter, respectively. FS originates from differences in the nuclear charge distributions due to the different number of neutrons and, to a good approximation, is proportional to the difference of rms charge radii $\delta\left\langle r^{2}\right\rangle_{a} \equiv$ $\left\langle r^{2}\right\rangle_{A_{0}}-\left\langle r^{2}\right\rangle_{A} . K_{i}, F_{i}$ and $X_{i}$ are constants that only depend on the electronic configurations associated with the transition $i$. This factorization of electronic and nuclear parameters can be understood from perturbation theory by noting that the nuclear mass is much larger than the electron mass $m_{e} / m_{A} \ll 1$, and

\footnotetext{
${ }^{1}$ Here we consider $A_{0}$ as the reference isotope, however, it also possible to preform the King analysis with neighboring isotope pairs.
} 
that only a small portion of the electron density penetrating the nucleus is sensitive to the finite nuclear size.

Whenever Eq. (1) is a valid description of the IS, it predicts that the so-called modified IS (mIS), $m v_{i}^{a} \equiv \mu_{a}^{-1} v_{i}^{a}$, for two distinct transitions $i=1,2$ are linearly related [23] in the absence of NP, $\alpha_{\mathrm{NP}}=0$. Indeed, the difference $\delta\left\langle r^{2}\right\rangle_{a}$ can be traded for a measurement in one transition as $\mu_{a}^{-1} \delta\left\langle r^{2}\right\rangle_{a}=$ $\left(m v_{1}^{a}-K_{1}\right) / F_{1}$, yielding for the second transition $m v_{2}^{a}=$ $F_{21} m v_{1}^{a}+K_{21}$, where $F_{21} \equiv F_{2} / F_{1}$ and $K_{21} \equiv K_{2}-F_{21} K_{1}$. This relation is known as King linearity. Note that knowledge of the nuclear charge distributions and the electronic constants $K_{i}$ and $F_{i}$ is not needed at all in order to make this prediction. It does, however, require determination of the nuclear masses with good accuracy.

New atomic forces typically break King linearity [22,24]. In the presence of NP, $\alpha_{\mathrm{NP}} \neq 0$, and repeating the steps above gives

$$
m v_{2}^{a}=F_{21} m v_{1}^{a}+K_{21}+\alpha_{\mathrm{NP}} X_{21} h_{a},
$$

where $X_{21} \equiv X_{2}-F_{21} X_{1}$ and $h_{a} \equiv \mu_{a}^{-1} \gamma_{a}$. The NP term thus induces NL, unless (i) the ratio $X_{i} / F_{i}$ is constant between the two transitions or (ii) $h_{a}$ is either independent of $a$ or $\propto m v_{1,2}^{a}$. The former arises when the range of the new force becomes shorter than the nuclear size. In this case, the atomic potentials for both FS and NP are described by a contact interaction and probe the same region of the electron cloud inside the nucleus, leading to $X_{i} \propto F_{i}$. Nevertheless, high sensitivity to the NP coupling $\alpha_{\mathrm{NP}}$ can be achieved away from these limits [24]. The NP coupling can be determined by solving Eq. (2) for three different isotope pairs $a_{1,2,3}$, yielding

$$
\alpha_{\mathrm{NP}}=\frac{\operatorname{det}\left(\vec{m} v_{1}, \vec{m} v_{2}, \vec{m} \mu\right)}{\operatorname{det}\left(X_{1} \vec{m} v_{2}-X_{2} \vec{m} v_{1}, \vec{h}, \vec{m} \mu\right)},
$$

where arrows denote vectors in the isotope space and $\overrightarrow{m \mu}=$ $(1,1,1)$. Note that the electronic constants $X_{1,2}$ and the nuclear parameter $\vec{h}$ of NP are the only theoretical inputs needed to extract $\alpha_{\mathrm{NP}}$ given a set of $\vec{m} v_{1,2}$ data. Equation (3) has a clear geometrical interpretation. The numerator on the righthand side is the amount of NL in the data, which is related to the area of the triangle formed by three points on the King plot, while the denominator corresponds to the amount of NL predicted by theory (for a coupling of unity, $\alpha_{\mathrm{NP}}=1$ ). Using Eq. (1) the latter writes $\left(F_{1} X_{2}-F_{2} X_{1}\right) \times \operatorname{det}\left(m \delta\left\langle\overrightarrow{r^{2}}\right\rangle, \vec{h}, \overrightarrow{m \mu}\right)$. The first factor shows the suppression due to the alignment of electronic FS and NP constants in the high mass limit. The second factor is the alignment of nuclear parameters. Note that for $\gamma_{a}=\Delta A=A_{0}-A$, which is typically the case for new spin-independent interactions, the NP and MS nuclear parameters are approximately aligned since $\vec{h} \simeq-A_{0}^{2}(\vec{m} \mu-$ $\left.\overrightarrow{\Delta A} / A_{0}\right)$ resulting for instance in a sensitivity suppression of $\mathcal{O}(20)$ for $\mathrm{Yb}$.

\section{KING ANALYSIS WITHOUT ISOTOPES MASS}

In this section we present a King-like analysis that does not rely on the isotope masses as input parameters. The interest of this option, which can be viewed as a special case of the generalized King method, see Sec. V, is that it is fully based on IS spectroscopy measurements, which have great potential for improvement (see, e.g., Ref. [27]).

The linear relation of Eq. (2) (in the absence of NP) holds for $m v_{i}^{a}$, but not for $v_{i}^{a}$. Therefore testing King linearity requires good knowledge of the isotope masses. The total mIS uncertainty as a function of uncertainties in the IS and mass measurements is,

$$
u[m v]=\sqrt{u[v]^{2}+\frac{m_{A}^{2} u\left[m_{A_{0}}\right]^{2}+m_{A_{0}}^{2} u\left[m_{A}\right]^{2}}{\left(m_{A_{0}}-m_{A}\right)^{2}}},
$$

where $u[x] \equiv \sigma[x] / x$, with $\sigma[x]$ denoting the standard deviation of observable $x$. Hence, isotope masses must be known better than IS by a factor $\mathcal{O}\left(m_{A} /\left(m_{A_{0}}-m_{A}\right)\right)$. Otherwise, the assessment of King linearity will be limited by the relatively poor knowledge of nuclear masses, resulting in a weaker sensitivity to NP forces.

Here we present a simple modification of the King linearity definition that does not rely on precise isotope mass determination. The idea is to use IS measurements in a third transition (with the same isotope pairs) in order to remove the MS nuclear parameter that is not sufficiently well known. Consider the case of three transitions. The MS and FS nuclear parameters, respectively $\mu_{a}$ and $\delta\left\langle r^{2}\right\rangle_{a}$, are extracted using Eq. (1) applied to two of the transitions, and then plugged in the theory prediction for the third one. This yields a linear relation among the IS of the three transitions, up to a NP contribution,

$$
v_{3}^{a}=f_{3}^{\alpha} v_{\alpha}^{a}+\alpha_{\mathrm{NP}}\left(X_{3}-f_{3}^{\alpha} X_{\alpha}\right) \gamma_{a},
$$

where sum over $\alpha=1,2$ is implicit and

$$
\left(f_{3}^{1}, f_{3}^{2}\right)=\left(K_{3}, F_{3}\right) \cdot\left(\begin{array}{ll}
K_{1} & F_{1} \\
K_{2} & F_{2}
\end{array}\right)^{-1} \text {. }
$$

Similarly to the usual King linearity, this prediction can be tested without any additional theory inputs by checking the consistency of $\operatorname{det}\left(\vec{v}_{1}, \vec{v}_{2}, \vec{v}_{3}\right)$ with zero. We refer to this method as the no-mass King (NMK) analysis.

In the presence of NP, we can extract $\alpha_{\mathrm{NP}}$ by measuring IS of three transitions and three isotope pairs upon using Eq. (5), which yields

$$
\alpha_{\mathrm{NP}}=\frac{\operatorname{det}\left(\vec{v}_{1}, \vec{v}_{2}, \vec{v}_{3}\right)}{\frac{1}{2} \epsilon_{i j k} \operatorname{det}\left(\vec{v}_{i}, \vec{v}_{j}, X_{k} \vec{\gamma}\right)}
$$

where the sum over the indices is implicit. As for the original King linearity case, the numerator of Eq. (7) is a measure of NL present in the data, while the denominator corresponds to the amount of NL predicted by theory for $\alpha_{\mathrm{NP}}=1$. Equation (1) allows to express it in terms of the MS and FS parameters as $\epsilon_{i j k} K_{i} F_{j} X_{k} \times \operatorname{det}\left(\vec{\mu}, \delta\left\langle\overrightarrow{r^{2}}\right\rangle_{a}, \vec{\gamma}\right)$, which makes manifest the limits where sensitivity to NP is suppressed due to the alignment of either electronic constants (as in the heavy NP mass limit) or nuclear parameters. The NP sensitivity of the NMK analysis of Eq. (7) and its numerical comparison with the original King plot analysis is discussed in Sec. VI for the case of $\mathrm{Yb}$. 


\section{NONLINEARITIES IN KING PLOTS FROM NUCLEAR EFFECTS}

Equation (1) is not a complete description of the IS, see, e.g., Ref. [36]. It is only approximate, valid at leading order in the variation of the nuclear mass and charge distribution. This is straightforward to understand from standard (timeindependent) perturbation theory in quantum mechanics. At first order in a small perturbation (such as a change in nuclear parameters) only the eigenvalues of the Hamiltonian are corrected while the associated eigenstates are unchanged. Hence, at first order in the variation of nuclear mass and charge distribution, the electronic configurations remain the same between isotopes, yielding the factorized form in Eq. (1). However, at second order in the perturbation, the electron cloud will respond to a change of the nuclear properties and make both MS and FS electronic constants isotope dependent, $K_{i} \rightarrow K_{i}^{a}$ and $F_{i} \rightarrow F_{i}^{a}$, which induces King linearity breaking.

Experimentally, there are very few observations of King plot NLs. Large NLs of $\mathcal{O}(10) \mathrm{MHz}$ were observed in samarium isotopes [37], which however was later understood to result from a strong enhancement of second-order FS effect due to mixing with a nearly-degenerate level [38,39]. A nonlinear behavior at the $100 \mathrm{kHz}$ level was recently reported in strontium [40]. However the King plot of Ref. [40] was constructed using ${ }^{87} \mathrm{Sr}$ which may indicate the presence of sizable effects from the nuclear spin of this odd isotope. Finally, we note that the very recent $3 \sigma$ evidence for $\mathrm{NL}$ in $\mathrm{Yb}^{+}$ quadrupole transitions [32] is consistent with an interpretation in terms of second-order FS or may be an indication of NP.

Calculating the NLs induced by nuclear effects in a given element is theoretically challenging. The main difficulty arises from the fact that most of the higher order corrections are aligned with the leading order contributions in Eq. (1) and therefore they do not yield NLs. A well understood example is higher moments in the nuclear charge distribution [41]. The theoretical evaluation of NLs in King plots was reconsidered in Ref. [30] which presented a first-time calculation of King plot NLs for narrow transitions in several heavy elements where the dominant contribution was found to arise from second order FS.

With improvements in the experimental accuracy, additional nuclear parameters, such as higher moments of the charge distribution, may become relevant. Other subleading effects include nuclear polarizability $[42,43]$ and quadratic mass shift [44]. As pointed out in Ref. [35] these effects are formally parameterized, without loss of generality, by adding to Eq. (1) an infinite series of terms involving new nuclear parameters different from $\mu_{a}$ and $\delta\left\langle r^{2}\right\rangle_{a}$,

$$
\nu_{i}^{a}=K_{i} \mu_{a}+F_{i} \delta\left\langle r^{2}\right\rangle_{a}+\sum_{l=2}^{\infty} F_{i l} \lambda_{l, a},
$$

where $\lambda_{l, a}$ are independent nuclear parameters and $F_{i l}$ are electronic constants. Once experimental precision in IS measurement reaches the level of the (dominant) subleading effects, SM NLs arise in King plots and it becomes challenging to probe NP.

A possible strategy would be to calculate those NLs from atomic and/or nuclear theory, subtract them from the mea- surements, and construct a King plot with the residual mIS. However, as already emphasized, such calculations are rather challenging. Moreover, to understand the level at which the SM nonlinearity will be observed, the calculation of an isotope invariant measure, like Eq. (18) below, is needed.

Another strategy, where two transitions and four isotope pairs are available is presented in Ref. [32]. Instead of constructing a King plot of the two mIS, they considered the correlation between one transition and $\mu_{a}$, normalized by the second transition, which is predicted to be linear by the SM at leading order. The leading SM source of NL and NP can be distinguished if prior knowledge about the former is included, for instance if originating from second order FS, so that a particular pattern of NLs can be predicted. Then, provided measurements (and theoretical inputs) are accurate enough, one can distinguish between the two effects.

\section{GENERALIZED KING LINEARITY AND NEW PHYSICS}

Here we further extend the generalized King plot analysis of Ref. [35], such that physics beyond the SM could still be probed in the presence of NLs in King plots using minimal theoretical inputs. The idea is to use IS measurements of additional transitions in order to fix the nuclear and electronic parameters that break King linearity at leading order.

IS are small perturbations to the atomic spectra, with effects scaling like ratios of nuclear scales to atomic scales. Hence, the number of $\lambda_{l, a}$ that are relevant given a fixed experimental precision is expected to be finite and (hopefully) small. The exact number of such parameters and their nuclear structure, i.e., their isotope dependence, could for instance be inferred from atomic theory calculations, see, e.g., Ref. [30].

In the current analysis, we assume $l=2, \ldots, m-1$, where $m$ is (at most) equal to the number clock transitions available for a given element, both neutral and charged counterparts. Then, all mIS for that element depend on $m-1$ independent nuclear parameters,

$$
m v_{i}^{a}=K_{i}+\sum_{l=1}^{m-1} F_{i l} m \lambda_{l, a}+\alpha_{\mathrm{NP}} X_{i} h_{a},
$$

where for convenience we have defined $F_{i 1} \equiv F_{i}, \lambda_{1, a}=$ $\delta\left\langle r^{2}\right\rangle_{a}$, and $m \lambda_{l, a} \equiv \mu_{a}^{-1} \lambda_{l, a}$. Hence we need at least $m-1$ transitions to fix the unknown nuclear parameters

$$
m \lambda_{l, a}=\left(F^{-1}\right)_{l i}\left(m v_{i}^{a}-K_{i}-\alpha_{\mathrm{NP}} X_{i} h_{a}\right),
$$

where $F^{-1}$ denotes the inverse of the matrix $F$ with $i, l=$ $1, \ldots, m-1$, and there is an implied sum over $i$. Consequently, the mIS for any other independent transition $k \neq$ $1, \ldots, m-1$ is predicted to be linearly related to the previous $m-1$ ones, up to a linearity-breaking term from NP

$$
m v_{k}^{a}=\left[K_{k}-f_{k i} K_{i}\right]+f_{k i} m v_{i}^{a}+\alpha_{\mathrm{NP}}\left[X_{k}-f_{k i} X_{i}\right] h_{a},
$$

where $k \neq i$ and $f_{k i} \equiv F_{k l}\left(F^{-1}\right)_{l i}$, where $l \neq k$ in the sum. The interpretation of the above expression is straightforward. In the absence of NP, the modified isotope shifts for $m$ transitions lie on a $(m-1)$-dimensional subspace. Consequently, $n=$ $m+1$ independent isotope pairs (points on the generalized $m$-dimensional King plot), thus $m+2$ isotopes, are needed in order to experimentally test this prediction. In cases where 


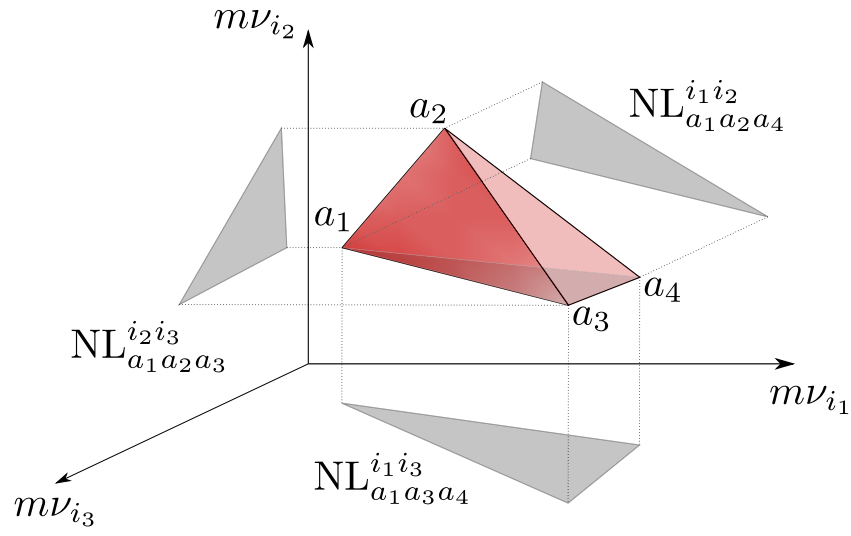

FIG. 1. Schematic illustration of the volume of nonlinearities (red) in the space of modified IS, as defined in Eq. (12) for the special case of $m=3$ transitions and $n=4$ isotope pairs. Gray triangles represent the amount of nonlinearities obtained in 2D King plots, as defined in Eq. (18), and are projections of three independent tetrahedron's faces on the corresponding two-dimensional IS subspace.

the system of equations is overconstrained, that is when there are more measurements than parameters to be determined, then one may consider fitting methods like those presented in Refs. [25,33]. A given data set is planar if the volume of the $m$-dimensional parallelotope formed by the $m$ measured $\vec{m} v$ vectors (each of them being of $n$ dimensions, the number different isotope pairs $a_{1}, \ldots, a_{n}$ ) is consistent with zero within experimental errors. Such a volume is given by the fully antisymmetric product of the measured $\vec{m} v$ 's and the unit vector in isotope space $\overrightarrow{m \mu}=(1, \ldots, 1)$,

$$
V=\operatorname{det}\left(\vec{m} v_{1}, \ldots, \vec{m} v_{m}, \vec{m} \mu\right) .
$$

Such a volume is illustrated in Fig. 1 in the case of $m=3$ transitions and $n=4$ independent isotope pairs. The volume induced by the theory ansatz in Eq. (11) is found to be

$$
V_{\text {th }}=\frac{\alpha_{\mathrm{NP}}}{(n-2) !} \epsilon_{i_{2}, \ldots, i_{n}} \epsilon_{a_{1}, \ldots, a_{n}} 1_{a_{1}} X_{i_{2}} h_{a_{2}} m v_{i_{3}}^{a_{3}} \ldots m v_{i_{n}}^{a_{n}},
$$

with the transition indices $i_{1}, \ldots, i_{n-1}$ and the isotope pairs indices $a_{1}, \ldots, a_{n}$ are taking values in $1, \ldots, n-1$ and $1, \ldots, n$, respectively. Therefore the NP coupling is given by

$$
\alpha_{\mathrm{NP}}=\frac{V}{V_{\mathrm{th}}\left(\alpha_{\mathrm{NP}}=1\right)} .
$$

We learn that even if the original notion of King linearity breaks down, NP can be probed. In the following, we refer to this method as generalized King $(G K)$ analysis.

In order to understand the limitations of the GK method, we write $V_{\text {th }}$ in terms of the different nuclear and electronic parameters

$$
V_{\mathrm{th}}=\operatorname{det}\left(F_{i l}, X_{i}\right) \operatorname{det}\left(h_{a}, m \mu_{a}, m \lambda_{l, a}\right) .
$$

Therefore we can see that if any of the two parameters are aligned, the NP sensitivity is lost.

Let us consider the case of four IS pairs and three transitions, i.e., $n=4$ and 3. The uncertainty in $\alpha_{\mathrm{NP}}$ can be derived by usual first-order error propagation from Eq. (14), $\sigma\left[\alpha_{\mathrm{NP}}\right]=$ $\sqrt{\sum_{k}\left(\partial \alpha_{\mathrm{NP}} / \partial \mathcal{O}_{k}\right)^{2} \sigma[k]^{2}}$, where $k$ runs over all experimental observables $\mathcal{O}_{k}$. Under the assumption that only $\sigma\left[v_{i}^{a}\right] \neq 0$, it can be written in terms of the 2D King plot NLs

$$
\sigma\left[\alpha_{\mathrm{NP}}\right]=\sqrt{\sum_{a, l}\left(\frac{\sigma\left[v_{i}^{a}\right]}{\mu_{a}} \sigma_{\alpha_{\mathrm{NP}}}^{a l}\right)^{2}},
$$

with

$$
\sigma_{\alpha_{\mathrm{NP}}}^{a l}=12 \frac{\epsilon_{i j k} X_{i} \mathrm{NL}_{\bar{a}}^{j k} \epsilon_{a_{1} a_{2} a_{3} a_{4}} h_{a_{1}} \mathrm{NL}_{a_{2} a_{3} a_{4}}^{\bar{l}}}{\left(\epsilon_{i j k} \epsilon_{a_{1} a_{2} a_{3} a_{4}} X_{i} h_{a 1} \mathrm{NL}_{a_{2} a_{3} a_{4}}^{j k}\right)^{2}},
$$

where the $\bar{a}$ is a $3 \mathrm{D}$ vector in the isotope space excluding the isotope pair $a, \bar{l}$ is a $2 \mathrm{D}$ vector in the transition space excluding the transition $l$ and

$$
\mathrm{NL}_{a_{1} a_{2} a_{3}}^{i j}=\left(\vec{m} v_{i} \times \vec{m} v_{j}\right) \cdot \vec{m} \mu,
$$

with the vectors $\vec{m} v_{i}$ in the isotope space built from the isotope pairs $a_{1}, a_{2}$ and $a_{3}$.

Finally, we emphasize that the GK analysis above can be straightforwardly repeated in cases having a different combination of known and unknown nuclear parameters. Also, while here we assumed that the isotope mass uncertainties were irrelevant, that is nuclear masses were known with sufficient precision, the latter can always be replaced by adding one more measured transition as in the NMK analysis in Sec. III.

\section{SENSITIVITY TO NEW PHYSICS: THE YTTERBIUM CASE}

In this section, we demonstrate the potential of the NMK and GK analyses presented in Secs. III and V in probing NP forces. We focus on the case of a new spin-independent interaction mediated by a force carrier $\phi$ of $\operatorname{spin} s=0,1$, and 2 . The effective Yukawa potential associated with the new intra-atomic force is given by $V_{\phi}(r)=-\alpha_{\mathrm{NP}}(A-Z) e^{-m_{\phi} r} / r$, where $m_{\phi}$ is the force carrier mass and $\alpha_{\mathrm{NP}}=(-1)^{s} y_{e} y_{n} / 4 \pi$, with $y_{e}\left(y_{n}\right)$ is the $\phi$-electron ( $\phi$-neutron) coupling and $\gamma_{a}=$ $A_{0}-A$. Note that we gross over a potential $\phi$-proton coupling here, since its effect would cancel in the IS. We also assume that there are no cancellation between IS contributions from the SM and NP. This is reasonable assumption since known $\mathrm{SM}$ effects do not scale like the atomic number difference $A_{0}-A$

Systems with several (measurable) optical clock transitions are quite rare. Ytterbium is a rather unique element where three such transitions have been observed: two in the $\mathrm{Yb}^{+}$ ion, $4 f^{14} 6 s\left({ }^{2} S_{1 / 2}\right) \rightarrow 4 f^{14} 5 d\left({ }^{2} D_{3 / 2}\right)$ and $4 f^{14} 6 s\left({ }^{2} S_{1 / 2}\right) \rightarrow$ $4 f^{13} 6 s^{2}\left({ }^{2} F_{7 / 2}\right)$, and one in neutral $\mathrm{Yb}, 4 f^{14} 6 s^{2}\left({ }^{1} S_{0}\right) \rightarrow$ $4 f^{14} 6 s 6 p\left({ }^{3} P_{0}\right)$, at a wavelength of 436,467 , and $578 \mathrm{~nm}$, respectively. The parenthesis indicates the term symbol of the angular momentum quantum number associated with the multielectron state. For simplicity, in the following, we refer to the above transitions as $S-D_{3 / 2}, S-F$, and $S-P$, respectively. The three of them have been measured with sub-Hz precision for the $A=171$ isotope [45-48]. Another candidate clock transition in neutral $\mathrm{Yb}$, yet to be measured with high precision, is $4 f^{14} 6 s 6 p\left({ }^{3} P_{0}\right) \rightarrow 4 f^{13} 6 s^{2} 5 d J=2$ at $1696 \mathrm{~nm}$ [49]. Moreover, ytterbium has five stable isotopes with zero nuclear spin, $A=168,170,172,174$, and 176 and one 
TABLE I. The estimated SM 2D NL relative to the uncertainty, $\mathcal{R}_{\mathrm{NL}}^{\mathrm{SM}}$ [see Eq. (19)], for different combination of transitions and isotopes with respect to $A_{0}=174$.

\begin{tabular}{lcccc}
\hline \hline Transitions & \multicolumn{4}{c}{$\left|\mathcal{R}_{\mathrm{NL}}^{\mathrm{SM}}\right|\left(\mathrm{kHz} / \sigma_{v}\right)$} \\
\cline { 2 - 5 } Isotopes & $170,172,176$ & $168,172,176$ & $168,170,176$ & $168,170,172$ \\
\hline$S-F, S-D_{3 / 2}$ & 1.8 & 1.8 & 0.52 & 1.7 \\
$S-P, S-D_{3 / 2}$ & 2.9 & 2.8 & 0.8 & 2.7 \\
$S-P, S-F$ & 4.6 & 4.4 & 1.3 & 4.2 \\
$S-D_{3 / 2}, S-D_{5 / 2}$ & 0.75 & 0.72 & 0.21 & 0.70 \\
\hline \hline
\end{tabular}

radioactive isotope $(A=166)$ with a half-life of $\approx 2.4$ days. Hence, in principle, generalized King linearity can be tested with very high precision in three-dimensional, or even fourdimensional, King plots combining clock transitions in $\mathrm{Yb}^{0}$ and in $\mathrm{Yb}^{+}$.

The SM leading correction to Eq. (1) in the $\mathrm{Yb}$ system is the second-order FS [30]. By matching this correction to Eq. (8), it can be written as a single term, $F_{i 2} \lambda_{2, a}$, with $\lambda_{2, a} \approx$ $\left(\delta\left\langle r^{2}\right\rangle_{a}\right)^{2}$. This correction contributes to the SM NLs of the usual King plot. We quantify the NL induced on the King plot as

$$
\mathcal{R}_{\mathrm{NL}} \equiv \frac{\mathrm{NL}_{a_{1} a_{2} a_{3}}^{i j}}{\sigma\left[\mathrm{NL}_{a_{1} a_{2} a_{3}}^{i j}\right]},
$$

where $\mathcal{R}_{\mathrm{NL}} \gtrsim \mathcal{O}(1)$ is a clear signal of King linearity breaking. The definition of Eq. (19) can be generalized to any type of King plot, see also Ref. [33].

In our numerical estimations below, we evaluate the electronic parameters, $K_{i}, F_{i}, F_{i 2}$ (the SM parameters) and $X_{i}\left(m_{\phi}\right)$ (the NP parameters as functions of $m_{\phi}$ ) by using configuration interaction (CI) implemented in AMBiT [50], see Appendix for details. The resulting estimates of the SM electronic parameters are reported in Table II. The nuclear parameters, such as the nuclear masses $\left(m_{A}\right)$ and the charge radius differences $\left(\delta\left\langle r^{2}\right\rangle_{a}\right)$ are taken from Refs. [29,51,52], respectively, and reported in Table III for completeness.

Then, we evaluate $\mathcal{R}_{\mathrm{NL}}^{\mathrm{SM}}$ for the three ytterbium transitions and a given choice of three isotope pairs formed by either of $A=168,170,172,176$, and the reference $A_{0}=174$. In addition, we also estimate the NL between the $S \rightarrow D_{3 / 2}$ and $S \rightarrow D_{5 / 2} \mathrm{Yb}^{+}$transitions that were measured in Ref. [32]. For simplicity, we assume a common error bar, $\sigma_{v}$, for all the transitions and neglect other sources of uncertainty, like nuclear mass uncertainty. The results are summarized in Table I. In particular, we observe that $\mathcal{R}_{\mathrm{NL}}^{\mathrm{SM}} \sim$ $\mathcal{O}(0.2-5) \mathrm{kHz} / \sigma_{v}$ from which we conclude that NLs from subleading SM effects become important once the accuracy of the IS measurements crosses the $\mathrm{kHz}$ level.

Finally, we estimate the reach of the NMK and GK analyses to probe a new electron-neutron Yukawa interaction, and compare those to the original 2D King (2DK) analysis with zero and finite isotope mass uncertainties. While bounds on NP resulting from an experimental IS analysis depend only on a minimal set of theory inputs, viz. the NP parameters $X_{i}$ and $\gamma_{a}$, but not on the SM parameters, the estimated NP reach requires however knowledge of the electronic and nuclear parameters. In principle, the SM parameters can be extracted by fitting the data. Here, instead, we use the estimates in Table II for the electronic constants. In constructing our estimates for the projected reach of the various King plot analyses, we consider the $S-F$ and $S-D_{3 / 2}$ narrow transitions in $\mathrm{Yb}^{+}$, and we use the $S-P$ transition of $\mathrm{Yb}^{0}$ whenever a third transition is needed. Concerning the isotopes, we choose $A_{0}=174$ and $A=170,172,176$, and $A=168$ whenever the analysis requires a fourth isotope pair. Motivated by the experimental results of Ref. [27], we derive projections under the assumption that the IS measurement accuracy for all transitions is $\sigma\left[v_{i}^{a}\right]=0.01 \mathrm{~Hz}$. For sake of completeness, we also show in Fig. 2 (top panels) the projections where the current state-ofthe-art accuracy at the $\mathrm{Hz}$ level is assumed.

We perform two sets of comparisons. The first set focuses on the effect of the nuclear mass uncertainties. Thus, in this case, we assume zero NLs from the $\mathrm{SM}, \mathrm{NL}_{\mathrm{SM}}=0$. We then compare the following three analyses: (1) the $2 \mathrm{DK}$ with $\sigma\left[m_{A}\right]=0,(2)$ the $2 \mathrm{DK}$ with $\sigma\left[m_{A}\right] \neq 0$, and (3) the NMK of Sec. III.

The second set focuses instead on dealing with large SM NLs, while neglecting nuclear mass uncertainties, that is we set $\sigma\left[m_{A}\right]=0$. We then compare the following three analyses: (1) the $2 \mathrm{DK}$ with $\mathrm{NL}_{\mathrm{SM}}=0$, (2) the $2 \mathrm{DK}$ with $\mathrm{NL}_{\mathrm{NP}} \ll \mathrm{NL}_{\mathrm{SM}} \approx 1.5 \times 10^{8} \mathrm{MHz}^{2} \mathrm{amu}^{2}$, and (3) the GK of Sec. V.

Figure 2 shows the expected 95\% confidence level (CL) bounds, i.e., $\alpha_{\mathrm{NP}}<2 \sigma\left[\alpha_{\mathrm{NP}}\right]$, of the above two sets of comparisons, along with the current IS bound from $\mathrm{Ca}^{+}$[33] and the $95 \% \mathrm{CL}$ interval preferred by the NP interpretation of the $\mathrm{Yb}^{+}$results of Ref. [32]. For sake of completeness, we also show existing bounds from 5 th force searches [53,54], electron-neutron scattering [55], neutron-nucleus scattering [56-59] combined with the electron magnetic moment [24], hydrogen-deuterium (HD) IS for the $1 S-2 S$ transition where the charge radius difference is determined by muonic spectroscopy (under the assumption of no NP in the muonic sector) [60] and globular cluster [61]. As pointed out in Ref. [62] reasonable doubt persists regarding the SN 1987A bound [63], therefore we chose to omit it. Note that in general astrophysical bounds have different systematics and large model dependence, thus, independent terrestrial probes are well motivated. For reference, we also mark the theoretical lines predicted by the relaxed-relaxion model of Ref. [10] and by an hypothetical scalar with a finite lifetime that can accommodate the recent KOTO results [10,64-66]. In the above two scenarios, the interaction of new light particle with the SM is trough Higgs mixing. We assume that the electron and neutron Yukawa Higgs couplings saturate the current experimental upper bounds see [22,67-70].

From the left panels of Fig. 2, we learn that the nuclear mass uncertainties, if kept at the current level, will become an important limitation in searching for NP with the usual King plot analysis once IS measurement accuracy crosses the $\mathrm{Hz}$ level. However, this can be overcome by using instead the NMK analysis provided accurate IS measurements of an additional narrow transition are possible. The NMK analysis shows sensitivity to NP similar to the original 2DK with zero mass uncertainties. This motivates the use of a purely spectroscopy-based approach which does not rely on modified IS, but rather on IS frequencies directly. 

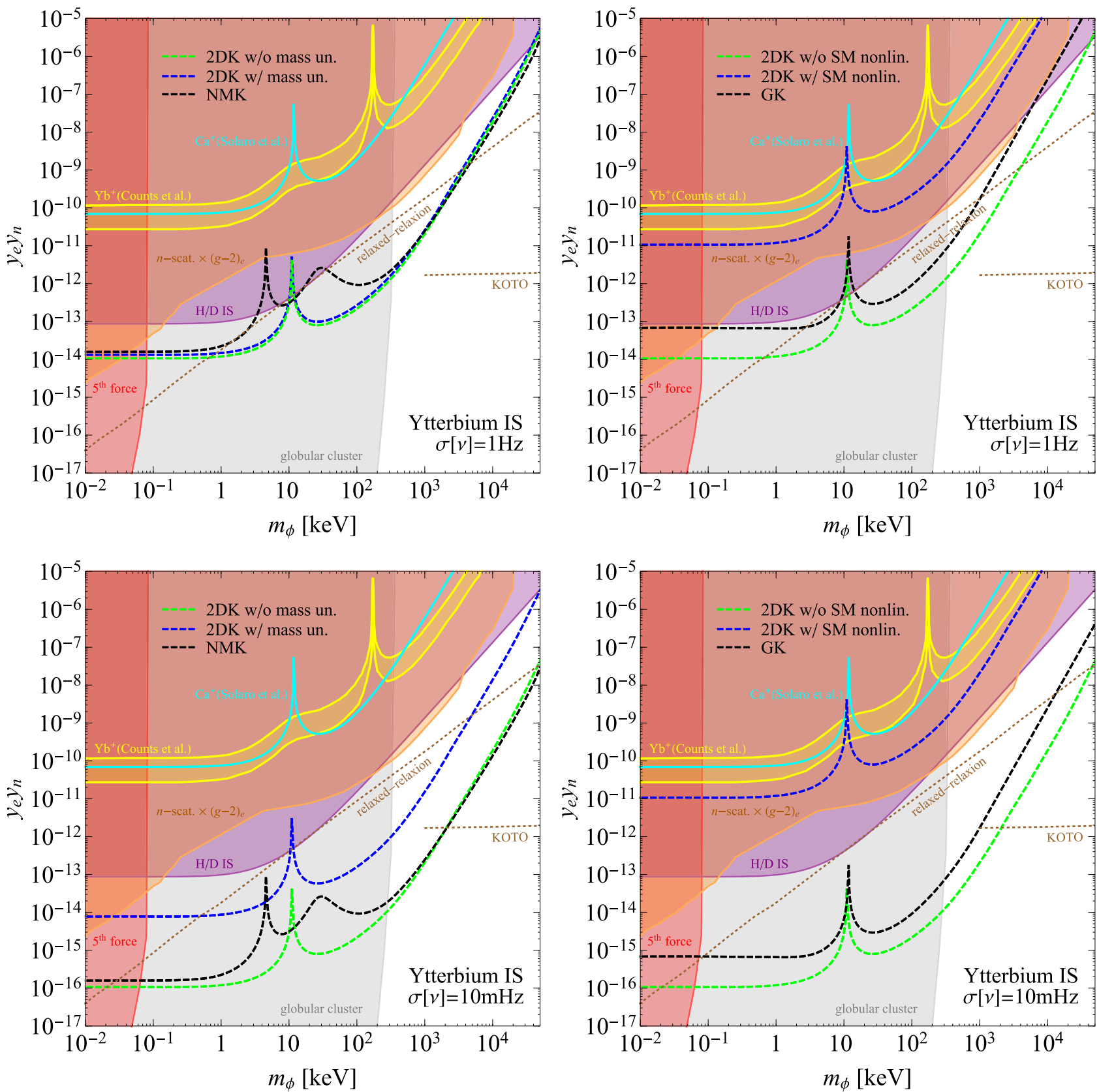

FIG. 2. The projected IS bounds in the Yb system (dashed lines) with a universal IS measurement uncertainty of $\sigma[v]=10 \mathrm{mHz}$ (bottom) and $1 \mathrm{~Hz}$ (top). (left) Comparison of projections from 2D King (2DK) analysis based on $S \rightarrow D_{3 / 2}$ and $S \rightarrow F$ transitions with zero non linearities (NLs) from the SM and omitting (green) or including (blue) current nuclear mass uncertainties, and from the no-mass King analysis (NMK) adding the $S \rightarrow P$ transition (black). (right) Comparison of projections from the 2DK analysis with zero nuclear mass uncertainty and including (blue) or not (green) $\mathrm{NL}_{\mathrm{SM}}$ and from the generalized King analysis (GK) adding the $S \rightarrow P$ transition (black). The existing NP bounds from IS spectroscopy in $\mathrm{Yb}^{+}$(the preferred 95\% CL NP interval of Ref. [32]) and $\mathrm{Ca}^{+}$(the upper bound from Ref. [33]) are shown in yellow and cyan solid lines, respectively. Also shown are the current bounds from fifth force searches [53,54], electron-neutron scattering [55], neutron-nucleus scattering [56-59] combined with the electron magnetic moment [24], 1S-2S hydrogen-deuterium (HD) IS [60], and globular cluster [61]. The dotted lines indicate the relaxed relaxion and a hypothetical scalar that may explain the KOTO results.

The right panels of Fig. 2 demonstrate the importance of accounting for NLs emerging from the SM higher order contributions to the IS, on the one hand, and how it can potentially limit the sensitivity of the usual King plot analysis to probe NP, on the other hand. Nevertheless, as we argued in Sec. II, the leading NL contributions of the SM can be fitted from the data and the GK analysis shows sensitivity to NP that compares to the original 2DK analysis.

We emphasize that the projections of Fig. 2 are derived under the assumption that the data will be consistent with linearity. Thus, those can be viewed as the best case scenario or a milestone, motivating further theoretical exploration of subleading nonlinear effects in the SM given a particular 


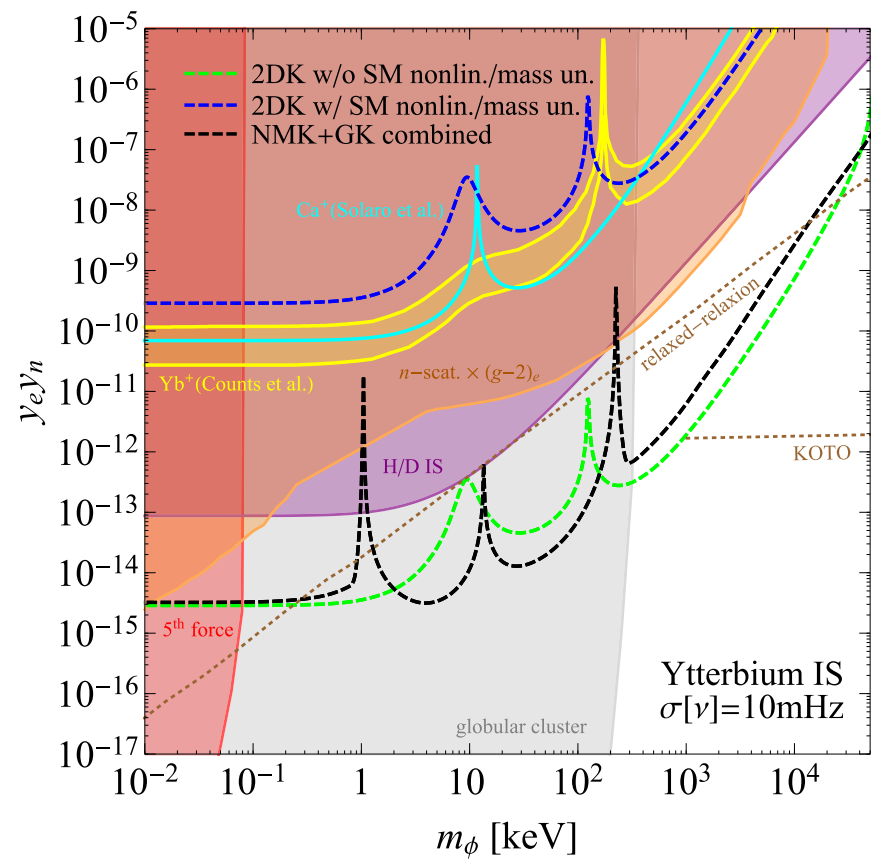

FIG. 3. The projected IS bounds in the Yb system (dashed lines) with a universal IS measurement uncertainty of $\sigma[v]=10 \mathrm{mHz}$. Comparison of projections from 2D King (2DK) analysis based on $S \rightarrow D_{3 / 2}$ and $S \rightarrow D_{5 / 2}$ transitions omitting (green) or including (blue) non linearities (NLs) from the SM and current nuclear mass uncertainties, and from a combined GK and NMK analysis adding the $S \rightarrow F$ and $S \rightarrow P$ transitions (black). The existing NP bounds from IS spectroscopy in $\mathrm{Yb}^{+}$(the preferred 95\% CL NP interval of Ref. [32]) and $\mathrm{Ca}^{+}$(the upper bound from Ref. [33]) are shown in yellow and cyan solid lines, respectively. Also shown are the current bounds from fifth force searches [53,54], electron-neutron scattering [55], neutron-nucleus scattering [56-59] combined with the electron magnetic moment [24], $1 S$-2S hydrogen-deuterium (HD) IS [60], and globular cluster [61]. The dotted lines indicate the relaxed relaxion and a hypothetical scalar that may explain the KOTO results.

experimental accuracy. For example, based on the estimates presented in Ref. [30], nuclear polarizability and the quadratic mass shift, whose scaling with neutron number differs from the NP contribution, are expected to induce NLs at the $\mathcal{O}(1) \mathrm{Hz}$ level in $\mathrm{Yb}^{+}$. In principle, future analyses may include additional theory inputs associated with such subleading effects, and in turn keep improving the NP sensitivity by further generalizing the three-dimensional GK analysis presented here.

The GK and NMK projections aim primarily at assessing how the limitations to the NP sensitivity can be overcome separately. Beyond this mere illustration purpose, each of them could still translate into actual bounds in the future provided the other one is taken care of by other means. For instance, the NMK method will be useful in the case that relevant SM NL contributions are known sufficiently well from theory. Conversely, the GK method will be useful in a situation where nuclear mass measurements are improved in the future. In the meantime, the GK and NMK methods could be easily combined if enough transitions and isotopes are available to experiments. This is illustrated in Fig. 3 where we compare the 2DK with $\sigma\left[m_{A}\right]=0$ and $\mathrm{NL}_{\mathrm{SM}}=0$, to the $2 \mathrm{DK}$ with $\sigma\left[m_{A}\right] \neq 0$ and $\mathrm{NL}_{\mathrm{NP}} \ll \mathrm{NL}_{\mathrm{SM}}$ and a combination of the GK and NMK methods using in addition the $S \rightarrow F$ transition in $\mathrm{Yb}^{+}$and the $S \rightarrow P$ one in neutral $\mathrm{Yb}$, and all of the five stable isotopes.

\section{CONCLUSIONS}

There have been some important developments in IS spectroscopy. In particular IS measurements recently reached an unprecedented level of accuracy of better than $10 \mathrm{mHz}$ in strontium [27]. This implies that the NP sensitivity with IS searches can potentially be improved by more than five orders of magnitude compared to the first NP-dedicated King analyses $[32,33]$. However, once entering in such a new regime of precision, experiments will quickly face two limitations from (i) the uncertainties in the nuclear mass determinations and (ii) the SM contributions to the NL of the King plot.

In this work, we present two data-driven methods to overcome them, namely the no-mass King analysis and the generalised King analysis, respectively. The proposed methods are based on the idea that more measurements of electronic transitions and IS allow us to determine additional nuclear and electronic parameters purely from the data without additional theory inputs. With enough transitions and isotope pairs, the above two methods can be combined into a single analysis which is insensitive to both the leading SM $\mathrm{NL}$ effect and the nuclear masses.

Finally, while the constraining power of both methods were demonstrated with ytterbium transitions, both can be applied equally well to other systems where the needed additional clock transitions may come from very different charged ion states [71].

\section{ACKNOWLEDGMENTS}

We thank V.V. Flambaum, E. Fuchs, R. Ozeri, G. Perez, S. Schiller, Y. Shpilman, and C. G. Solaro for fruitful discussions. J.C.B. is supported in this work by the Australian Research Council (DP190100974). C.D. is supported by the program Initiative d'Excellence of Grenoble-Alpes University under grant Contract Number ANR-15-IDEX-02. Y.S. is supported by the United States-Israel Binational Science Foundation (BSF) (NSF-BSF program Grant No. 2018683) and by the Azrieli Foundation. Y.S. is Taub fellow (supported by the Taub Family Foundation). This work was performed in part at Aspen Center for Physics, which is supported by National Science Foundation grant PHY-1607611.

\section{APPENDIX: DETAILS OF THE NUMERICAL CALCULATIONS}

The numerical atomic calculations in this work were performed using AMBiT [50]. For both neutral $\mathrm{Yb}$ and $\mathrm{Yb}^{+}$, the calculation begins with Dirac-Fock (relativistic Hartee-Fock) to obtain the self-consistent potential and core orbitals up to and including $5 s^{2} 5 p^{6} 4 f^{14}$. We then create the $6 s, 6 p$, and $5 d$ valence orbitals as eigenstates of the core Dirac-Fock potential. Higher-energy orbitals are generated by successively multiplying the orbitals by the radial function $r$ and 
TABLE II. The electronic parameters for the $\mathrm{Yb}$ and $\mathrm{Yb}^{+}$transitions relevant to this work, as estimated using the AMBiT code, see text for details.

\begin{tabular}{lccc}
\hline \hline Transition & $K_{i}(\mathrm{GHz} \mathrm{amu})$ & $F_{i}\left(\mathrm{MHz} / \mathrm{fm}^{2}\right)$ & $F_{i 2}\left(\mathrm{MHz} / \mathrm{fm}^{4}\right)$ \\
\hline$S-F$ & 5111 & 36218 & -201.12 \\
$S-D_{3 / 2}$ & -1040 & -14968 & 83.247 \\
$S-D_{5 / 2}$ & -1247 & -14715 & 81.908 \\
$S-P$ & -655 & -9719.2 & 54.277 \\
\hline \hline
\end{tabular}

orthogonalizing to the lower core and valence orbitals [72]. The $5 f$ orbital is generated by multiplying the $5 d$ orbital by $r$ and orthogonalizing to the $4 f$ core orbital. This procedure saturates the configuration interaction (CI) space more quickly than $B$ splines. We also found that the resulting values of $X_{i}$ are more stable in the high $m_{\phi}$ limit than when using $B$ splines, which we hypothesise may be due to small irregularities in the $B$ spline orbitals near the origin (see, e.g., Ref. [73] for a discussion of this topic). For this reason, we have not included MBPT in the current work (except to calculate the mass shift operators $K_{i}$, which are more sensitive to correlations and less sensitive to the wavefunction at the nucleus).

For the neutral $\mathrm{Yb}$ case we generate excited states up to $12 s p d f$. The configuration interaction includes all many-body basis states generated by single and double excitations from $6 s^{2}, 6 s 6 p, 6 s 5 d$, and $6 p^{2}$. We also allow single excitations from the core $5 s, 5 p$, and $4 f$ shells. The electronic parameters
TABLE III. The nuclear parameters of $\mathrm{Yb}$ relevant to this work. The nuclear masses $m_{A}=m_{A, \mathrm{Yb}^{0}}-70 m_{e}+E_{b}$, with $E_{b}$ the binding energy, are taken from Refs. [29,51]. Note that the mass uncertainty is only due to the uncertainty in the atomic mass $m_{A, \mathrm{Yb}^{0}}$ as the correlated errors on $m_{e}$ and $E_{b}$ are mostly canceled in $\mu_{a}$. The difference in the rms charge radii are taken from Ref. [52] and are relative to $A=176$, we neglect the associated errors.

\begin{tabular}{lccc}
\hline \hline Isotope & $m_{A}(\mathrm{amu})$ & $\sigma\left[m_{A}\right]\left(10^{-9} \mathrm{amu}\right)$ & $\delta\left\langle r^{2}\right\rangle_{a}\left(\mathrm{fm}^{2}\right)$ \\
\hline 168 & 167.8958990 & 1300 & -0.5406 \\
170 & 169.896777237 & 11 & -0.3845 \\
172 & 171.898396650 & 15 & -0.2366 \\
174 & 173.900877539 & 12 & -0.1159 \\
176 & 175.904584700 & 16 & - \\
\hline \hline
\end{tabular}

$X_{i}$ and $F_{i}$ are generated by adding the corresponding potential to the Hamiltonian and taking the difference.

The treatment of $\mathrm{Yb}^{+}$is similar, however since we are targeting the $4 f^{13} 6 s^{2}$ configuration the CI matrix grows much more quickly than in the neutral $\mathrm{Yb}$ case. We include excited states up to $8 s p d f$ and take single and double excitations from the configurations $4 f^{14} 6 s, 4 f^{14} 6 p, 4 f^{14} 5 d, 4 f^{13} 6 s^{2}$, $4 f^{13} 6 s 5 d, 4 f^{13} 6 p^{2}$, and $4 f^{13} 5 d^{2}$. We also limit the number of additional excitations from the $4 f$ shell to one.

Our numerical estimation for the electronic parameters are given in Table II. In addition Table III contains the nuclear parameters relevant to this work.
[1] A. Davidson and K. C. Wali, minimal Flavor Unification Via Multigenerational Peccei-Quinn Symmetry, Phys. Rev. Lett. 48, 11 (1982).

[2] F. Wilczek, Axions and Family Symmetry Breaking, Phys. Rev. Lett. 49, 1549 (1982).

[3] G. B. Gelmini, S. Nussinov, and T. Yanagida, Does Nature Like Nambu-Goldstone Bosons? Nucl. Phys. B 219, 31 (1983).

[4] J. E. Kim, Light pseudoscalars, particle physics and cosmology, Phys. Rep. 150, 1 (1987).

[5] J. L. Feng, T. Moroi, H. Murayama, and E. Schnapka, Third generation familons, $\mathrm{b}$ factories, and neutrino cosmology, Phys. Rev. D 57, 5875 (1998).

[6] P. W. Graham, D. E. Kaplan, and S. Rajendran, Cosmological Relaxation of the Electroweak Scale, Phys. Rev. Lett. 115, 221801 (2015).

[7] R. S. Gupta, Z. Komargodski, G. Perez, and L. Ubaldi, Is the relaxion an axion? J. High Energy Phys. 02 (2016) 166.

[8] T. Flacke, C. Frugiuele, E. Fuchs, R. S. Gupta, and G. Perez, Phenomenology of relaxion-Higgs mixing, J. High Energy Phys. 06 (2017) 050.

[9] C. Frugiuele, E. Fuchs, G. Perez, and M. Schlaffer, Relaxion and light (pseudo)scalars at the HL-LHC and lepton colliders, J. High Energy Phys. 10 (2018) 151.

[10] A. Banerjee, H. Kim, O. Matsedonskyi, G. Perez, and M. S. Safronova, Probing the relaxed relaxion at the luminosity and precision frontiers, J. High Energy Phys. 07 (2020) 153.

[11] J. S. M. Ginges and V. V. Flambaum, Violations of fundamental symmetries in atoms and tests of unifica- tion theories of elementary particles, Phys. Rep. 397, 63 (2004).

[12] S. G. Karshenboim, Precision physics of simple atoms: QED tests, nuclear structure and fundamental constants, Phys. Rep. 422, 1 (2005).

[13] M. S. Safronova, D. Budker, D. DeMille, D. F. J. Kimball, A. Derevianko, and C. W. Clark, Search for new physics with atoms and molecules, Rev. Mod. Phys. 90, 025008 (2018).

[14] V. Andreev et al. (ACME Collaboration), Improved limit on the electric dipole moment of the electron, Nature (London) 562, 355 (2018).

[15] J. Baron et al. (ACME Collaboration), Order of magnitude smaller limit on the electric dipole moment of the electron, Science 343, 269 (2014).

[16] D. Hanneke, S. Fogwell, and G. Gabrielse, New Measurement of the Electron Magnetic Moment and the Fine Structure Constant, Phys. Rev. Lett. 100, 120801 (2008).

[17] C. S. Wood, S. C. Bennett, D. Cho, B. P. Masterson, J. L. Roberts, C. E. Tanner, and C. E. Wieman, Measurement of parity nonconservation and an anapole moment in cesium, Science 275, 1759 (1997).

[18] J. Guena, M. Lintz, and M. A. Bouchiat, Measurement of the parity violating $6 \mathrm{~S}-7 \mathrm{~S}$ transition amplitude in cesium achieved within 2 x 10(-13) atomic-unit accuracy by stimulated-emission detection, Phys. Rev. A 71, 042108 (2005).

[19] C. Frugiuele, J. Pérez-Ríos, and C. Peset, Current and future perspectives of positronium and muonium spectroscopy as dark sectors probe, Phys. Rev. D 100, 015010 (2019). 
[20] K. Pachucki, V. Patkóš, and V. Yerokhin, Testing fundamental interactions on the helium atom, Phys. Rev. A 95, 062510 (2017).

[21] S. G. Karshenboim, Precise physics of simple atoms, in XVII International Conference on Atomic Physics; ICAP 2000, edited by P. De Natale, E. Arimondo, and M. Inguscio, AIP Conf. Proc. No. 551 (AIP, New York, 2001), p. 238.

[22] C. Delaunay, R. Ozeri, G. Perez, and Y. Soreq, Probing atomic Higgs-like forces at the precision frontier, Phys. Rev. D 96, 093001 (2017).

[23] W. H. King, Comments on the article "peculiarities of the isotope shift in the samarium spectrum", J. Opt. Soc. Am. 53, 638 (1963).

[24] J. C. Berengut et al., Probing New Long-Range Interactions by Isotope Shift Spectroscopy, Phys. Rev. Lett. 120, 091801 (2018).

[25] C. Frugiuele, E. Fuchs, G. Perez, and M. Schlaffer, Constraining new physics models with isotope shift spectroscopy, Phys. Rev. D 96, 015011 (2017).

[26] F. W. Knollmann, A. N. Patel, and S. C. Doret, Part-per-billion measurement of the $4^{2} S_{1 / 2} \rightarrow 3^{2} D_{5 / 2}$ electric quadrupole transition isotope shifts between ${ }^{42,44,48} \mathrm{Ca}^{+}$and ${ }^{40} \mathrm{Ca}^{+}$, Phys. Rev. A 100, 022514 (2019).

[27] T. Manovitz, R. Shaniv, Y. Shapira, R. Ozeri, and N. Akerman, Precision Measurement of Atomic Isotope Shifts using a Two-Isotope Entangled State, Phys. Rev. Lett. 123, 203001 (2019).

[28] G. Audi, F. G. Kondev, M. Wang, W. Huang, and S. Naimi, The NUBASE2016 evaluation of nuclear properties, Chin. Phys. C 41, 030001 (2017).

[29] M. Wang, G. Audi, F. G. Kondev, W. Huang, S. Naimi, and X. $\mathrm{Xu}$, The AME2016 atomic mass evaluation (II). tables, graphs and references, Chin. Phys. C 41, 030003 (2017).

[30] V. V. Flambaum, A. J. Geddes, and A. V. Viatkina, Isotope shift, nonlinearity of King plots, and the search for new particles, Phys. Rev. A 97, 032510 (2018).

[31] V. Yerokhin, R. Müller, A. Surzhykov, P. Micke, and P. Schmidt, Nonlinear isotope-shift effects in Be-like, B-like, and C-like argon, Phys. Rev. A 101, 012502 (2020).

[32] I. Counts, J. Hur, D. P. L. Aude Craik, H. Jeon, C. Leung, J. C. Berengut, A. Geddes, A. Kawasaki, W. Jhe, and V. Vuletić, Observation of $3 \sigma$ Nonlinear Isotope Shift in $\mathrm{Yb}^{+}$Search for New Boson, Phys. Rev. Lett. 125, 123002 (2020).

[33] C. Solaro, S. Meyer, K. Fisher, J. C. Berengut, E. Fuchs, and M. Drewsen, Improved Isotope-Shift-Based Bounds on Bosons Beyond the Standard Model Through Measurements of the ${ }^{2} \mathrm{D}_{3 / 2}-{ }^{2} \mathrm{D}_{5 / 2}$ Interval in $\mathrm{Ca}^{+}$, Phys. Rev. Lett. 125, 123003 (2020).

[34] F. Gebert, Y. Wan, F. Wolf, C. N. Angstmann, J. C. Berengut, and P. O. Schmidt, Precision Isotope Shift Measurements in Calcium Ions using Quantum Logic Detection Schemes, Phys. Rev. Lett. 115, 053003 (2015).

[35] K. Mikami, M. Tanaka, and Y. Yamamoto, Probing new intraatomic force with isotope shifts, Eur. Phys. J. C 77, 896 (2017).

[36] W. H. King, Isotope Shifts in Atomic Spectra (Springer Science \& Business Media, 2013).

[37] J. A. R. Griffith, G. R. Isaak, R. New, M. P. Ralls, and C. P. van $\mathrm{Zyl}$, Anomalies in the optical isotope shifts of samarium, J. Phys. B 12, L1 (1979).
[38] J. A. R. Griffith, G. R. Isaak, R. New, and M. P. Ralls, Anomalies in the optical isotope shifts of samarium, J. Phys. B 14, 2769 (1981).

[39] C. W. P. Palmer and D. N. Stacey, Theory of anomalous isotope shifts in samarium, J. Phys. B 15, 997 (1982).

[40] H. Miyake, N. C. Pisenti, P. K. Elgee, A. Sitaram, and G. K. Campbell, Isotope shift spectroscopy of the ${ }^{1} s_{0} \rightarrow{ }^{3} p_{1}$ and ${ }^{1} s_{0} \rightarrow{ }^{3} p_{0}$ transitions in strontium, Phys. Rev. Research $\mathbf{1}$, 033113 (2019).

[41] E. C. Seltzer, K X-Ray isotope shifts, Phys. Rev. 188, 1916 (1969).

[42] A. Migdal, Quadrupole and dipole gamma-radiation of nuclei, Zh. Eksp. Teor. Fiz. 15, 81 (1945).

[43] J. Levinger, Migdal's and Khokhlov's calculations of the nuclear photoeffect, Phys. Rev. 107, 554 (1957).

[44] C. Palmer, Reformulation of the theory of the mass shift, J. Phys. B 20, 5987 (1987).

[45] C. Tamm, S. Weyers, B. Lipphardt, and E. Peik, Stray-fieldinduced quadrupole shift and absolute frequency of the 688$\mathrm{THz}^{171} \mathrm{Yb}^{+}$single-ion optical frequency standard, Phys. Rev A 80, 043403 (2009).

[46] N. Huntemann, B. Lipphardt, C. Tamm, V. Gerginov, S. Weyers, and E. Peik, Improved Limit on a Temporal Variation of $m_{p} / m_{e}$ from Comparisons of $\mathrm{Yb}^{+}$and $\mathrm{Cs}$ Atomic Clocks, Phys. Rev. Lett. 113, 210802 (2014).

[47] R. Brown et al., Hyperpolarizability and Operational Magic Wavelength in an Optical Lattice Clock, Phys. Rev. Lett. 119, 253001 (2017).

[48] M. Schioppo, R. C. Brown, W. F. McGrew, N. Hinkley, R. J. Fasano, K. Beloy, T. H. Yoon, G. Milani, D. Nicolodi, J. A. Sherman, N. B. Phillips, C. W. Oates, and A. D. Ludlow, Ultrastable optical clock with two cold-atom ensembles, Nat. Photonics 11, 48 (2017).

[49] M. Safronova, S. Porsev, C. Sanner, and J. Ye, Two Clock Transitions in Neutral $\mathrm{Yb}$ for the Highest Sensitivity to Variations of the Fine-Structure Constant, Phys. Rev. Lett. 120, 173001 (2018).

[50] E. Kahl and J. Berengut, ambit: A programme for highprecision relativistic atomic structure calculations, Comput. Phys. Commun. 238, 232 (2019).

[51] A. Kramida, Y. Ralchenko, and J. Reader, Nist atomic spectra database (version 5.6.1), https://www.nist.gov/pml/atomicspectra-database.

[52] I. Angeli and K. Marinova, Table of experimental nuclear ground state charge radii: An update, At. Data Nucl. Data Tables 99, 69 (2013)

[53] M. Bordag, U. Mohideen, and V. M. Mostepanenko, New developments in the Casimir effect, Phys. Rept. 353, 1 (2001).

[54] M. Bordag, G. L. Klimchitskaya, U. Mohideen, and V. M. Mostepanenko, Advances in the Casimir effect, Int. Ser. Monogr. Phys. 145, 1 (2009).

[55] S. L. Adler, R. F. Dashen, and S. B. Treiman, Comments on proposed explanations for the $\mu$ - Mesic atom x-ray discrepancy, Phys. Rev. D 10, 3728 (1974).

[56] R. Barbieri and T. E. O. Ericson, Evidence Against the Existence of a Low Mass Scalar Boson from Neutron-Nucleus Scattering, Phys. Lett. B 57, 270 (1975).

[57] H. Leeb and J. Schmiedmayer, Constraint on Hypothetical Light Interacting Bosons from Low-Energy Neutron Experiments, Phys. Rev. Lett. 68, 1472 (1992). 
[58] V. V. Nesvizhevsky, G. Pignol, and K. V. Protasov, Neutron scattering and extra short range interactions, Phys. Rev. D 77, 034020 (2008).

[59] Yu. N. Pokotilovski, Constraints on new interactions from neutron scattering experiments, Phys. Atom. Nucl. 69, 924 (2006).

[60] C. Delaunay, C. Frugiuele, E. Fuchs, and Y. Soreq, Probing new spin-independent interactions through precision spectroscopy in atoms with few electrons, Phys. Rev. D 96, 115002 (2017).

[61] E. Hardy and R. Lasenby, Stellar cooling bounds on new light particles: Plasma mixing effects, J. High Energy Phys. 02 (2017) 033.

[62] N. Bar, K. Blum, and G. D'amico, Is there a supernova bound on axions? Phys. Rev. D 101, 123025 (2020).

[63] G. Raffelt, Limits on a CP-violating scalar axion-nucleon interaction, Phys. Rev. D 86, 015001 (2012).

[64] T. Kitahara, T. Okui, G. Perez, Y. Soreq, and K. Tobioka, New Physics Implications of Recent Search for $K_{L} \rightarrow \pi^{0} v \bar{v}$ at KOTO, Phys. Rev. Lett. 124, 071801 (2020).

[65] S. Shinohara, Search for the rare decay $K_{L} \rightarrow \pi^{0} v \bar{v}$ at J-PARC KOTO experiment, https://indico.cern.ch/event/ 769729/contributions/3510939/attachments/1904988/3145907/ KAON2019_shinohara_upload.pdf. KAON2019, Perugia, Italy, 10-13 September 2019.
[66] P. B. Dev, R. N. Mohapatra, and Y. Zhang, Constraints on long-lived light scalars with flavor-changing couplings and the KOTO anomaly, Phys. Rev. D 101, 075014 (2020).

[67] G. Perez, Y. Soreq, E. Stamou, and K. Tobioka, Constraining the charm Yukawa and Higgs-quark coupling universality, Phys. Rev. D 92, 033016 (2015).

[68] Y. Zhou, Constraining the Higgs boson coupling to light quarks in the $\mathrm{H} \rightarrow \mathrm{ZZ}$ final states, Phys. Rev. D 93, 013019 (2016).

[69] A. Dery, C. Frugiuele, and Y. Nir, Large Higgs-electron Yukawa coupling in 2HDM, J. High Energy Phys. 04 (2018) 044.

[70] W. Altmannshofer, J. Brod, and M. Schmaltz, Experimental constraints on the coupling of the Higgs boson to electrons, J. High Energy Phys. 05 (2015) 125.

[71] P. Micke, T. Leopold, S. A. King, E. Benkler, L. J. Spieß, L. Schmöger, M. Schwarz, J. R. Crespo López-Urrutia, and P. O. Schmidt, Coherent laser spectroscopy of highly charged ions using quantum logic, Nature (London) 578, 60 (2020).

[72] P. Bogdanovich and G. Žukauskas, Approximate allowance for superposition of configurations in atomic spectra, Sov. Phys. Collect 23, 13 (1983).

[73] K. Beloy and A. Derevianko, Application of the dual-kineticbalance sets in the relativistic many-body problem of atomic structure, Comput. Phys. Commun. 179, 310 (2008). 